\title{
Aspectos anatômicos das artérias renais em queixadas (Tayassu pecari Link, 1795)
}

\section{Anatomical aspects of the renal arteries in white-lipped peccaries (Tayassu pecari Link,1795)}

\author{
G. V. Machado, ${ }^{\star}$ A. B. Mello, ${ }^{\star \star}$ O. Cunha, ${ }^{\star *}$ E. S. Bertoni, ${ }^{\star *}$ M. A. M. Carvalho***
}

\begin{abstract}
Resumo
Utilizaram-se 30 pares de rins de queixadas (Tayassu pecari), obtidos de animais adultos, machos e fêmeas, 15 dos quais tiveram suas artérias injetadas com solução corada de Neoprene Látex "450". Em seguida, foram fixados em solução aquosa de formol a $10 \%$ e, posteriormente, dissecados. Os outros 15 pares foram injetados pela mesma via arterial, agora com Vinilite dissolvido em acetona e corado, em seguida submetidos à corrosão ácida, visando o reconhecimento do padrão vascular arterial renal, particularmente no referente às divisões e subdivisões das artérias renais. Os resultados obtidos permitem afirmar que: a) as artérias renais, sempre únicas, dividem-se, aos níveis pré-hilar e hilar renais, em dois ou três ramos setoriais; b) os ramos setoriais da artéria renal direita, quando duplos, apresentam-se como dorsal e ventral (36,6\%) ou cranial e caudal (16,6\%); quando triplos, esses ramos se dispõem como ventral, dorsocranial e dorsocaudal (36,6\%); c) os ramos setoriais da artéria renal esquerda, quando duplos, exibem-se como dorsal e ventral $(33,3 \%)$ ou cranial e caudal $(16,6 \%)$; quando triplos, esses ramos ora são ventral, dorsocranial e dorsocaudal (36,6\%), ora dorsal, ventrocranial e ventrocaudal (13,3\%); d) os ramos setoriais, por sua vez, emitem de dois a três ramos segmentares destinados a territórios próprios do parênquima renal.
\end{abstract}

Palavras-chave: Tayassuidae; Tayassu pecari, anatomia; artérias renais.

\begin{abstract}
It was studied 30 pairs of kidneys of withe-lipped peccaries (Tayassu pecarı), obtained of adult animals, males and females, 15 of the which had its arteries injected with red-faced solution of Neoprene Latex "450", soon after they were fastened in aqueous solution of formalin at $10 \%$ and later it was dissected. The other 15 pairs were injected, by the renal arteries, now with Vinilite dissolved in acetone and red-faced, soon after submitted to the acid corrosion, seeking the recognition of the renal arterial vascular pattern, particularly in the regarding the divisions and subdivisions of the renal arteries. The obtained results allow to affirm that: a) the renal arteries, always only, they divide, to the levels pre-hilar and hilar renal, in two or three sectorial branches; b) the sectorial branches of the right, when double, come as dorsal and ventral (36.6\%) or cranial and caudal (16.6\%); when triples, those branches are disposed as ventral, dorsocranial and dorsocaudal $(36.6 \%)$; $\mathrm{c}$ ) the sectorial branches of the left renal artery, when double, are exhibited as dorsal and ventral (33.3\%) or cranial and caudal (16.6\%); when triples, those branches are ventral, dorsocranial and dorsocaudal (36.6\%) or dorsal, ventrocranial and ventrocaudal (13.3\%); d) the sectorial branches, for its time, emit of two to three segmentary branches destined to territories of the kidneys.
\end{abstract}

Keywords: Tayassuidae; Tayassu pecari; anatomy; renal arteries.

\section{Introdução}

O queixada (Tayassu pecari) pertence à subordem dos Suiformes, que compreende os Artiodáctilos de membros curtos e formas maciças, que não ruminam. Pertence à Família dos Tayassuidae, estes exclusivamente habitantes das Américas, podendo ser encontrados desde o Sul dos EUA até a Patagônia. Esse animal vem assumindo importância crescente no âmbito da oferta alimentar alternativa para o homem, tendo em vista que sua carne é tida como de alto teor protéico e baixo colesterol, acrescido da facilidade de adaptação ao cativeiro e baixo índice de competição na cadeia alimentar do homem. No Brasil, já se encontram vários criadouros autorizados desses animais, e sua carne já vem sendo ofertada em restaurantes especializados. O comportamento do queixada, assim como sua biologia e distribuição geográfica, foram descritos por Langer (1979), Kiltie (1981), Orr (1986) e Fowler (1986).

Tendo em vista a escassez de informações referentes à morfologia dos taiassuídeos, em particular do queixada, implantou-se uma linha de pesquisa, no Laboratório de Anatomia Veterinária da UFPR/Campus Palotina, direcionada à busca de conhecimentos referentes à anatomia desses animais.

\footnotetext{
* Professor de Anatomia Veterinária da UFPR/Campus Palotina - CEP 85950-000 - Palotina,PR.

** Estudante/Bolsista de Iniciação Científica/CNPq.

*** Professora de Anatomia Veterinária da UFPi.
} 
Além de oferecer subsídios ao estudo da anatomia comparativa, ao acrescentar dados que ampliem as informações sobre a espécie em foco, o presente trabalho tem por pressuposto a ampliação do conhecimento sobre importante representante da fauna brasileira. Nesta oportunidade, buscaramse dados referentes ao comportamento vascular arterial nos rins dessa espécie.

O tema em questão vem recebendo destaque proporcional à ampliação do interesse da cirurgia experimental, particularmente das ressecções parciais de órgãos, que colocam em pauta a questão da segmentação anatômica. Vale lembrar que, em anatomia, segmento é um território ou parte de um órgão com função, suprimento e drenagem sangüíneas independentes (Didio, 1985). Entretanto, os compêndios de Anatomia Veterinária mostram-se bastante sucintos e mesmo incompletos ao cogitarem da matéria. Ao tratarem dos vasos renais, no cavalo, Caradonna (1930), Favilli (1931) e Bossie (s. d.) referem-se à penetração de dois ou três ramos da artéria renal, ao passo que Ellemberger, Baum (1932) destacam a presença de quatro ou cinco ramos, sempre acompanhados de uma veia. Lesbre (1923), Bruni, Zimmerl (1947), no entanto, fazem alusão a vários ramos hilares, oriundos da artéria renal, a penetrar no órgão. Já Schummer, Nickel (1960), Schwarze, Schröder (1962), Dobberstein, Hoffmann (1963) afirmam que a artéria renal atinge o hilo indivisa, enquanto a veia renal desponta já constituída. Miglino et al. (1985) encontraram, em carneiros, de quatro a onze ramos hilares, os quais foram dispostos em quadrantes cranioventral, craniodorsal, caudoventral e caudodorsal. Porém El-Khaligi et al. (1987), nessa mesma espécie, considerando apenas as divisões primárias das artérias renais, referem-se a três ramos: cranial, caudodorsal e caudoventral. No referente às divisões primárias das artérias renais fica evidente, pelas exposições de Peduti-Neto (1976), Albuquerque (1979), Miglino et al. (1985), Carvalho, Pereira (1987), Carvalho (1995), todos em pequenos ruminantes, sua divisão em ramos dorsal e ventral. Porém, em suínos, essas divisões são predominantemente em ramos cranial e caudal, conforme Ferreira (1975), Fagundes (1984), Sampaio et al. (1996), Evan et al. (1996), Souza (1997).

\section{Material e métodos}

Com o intuito de colher informações referentes ao padrão vascular arterial dos rins do queixada (Tayassu pecari), utilizaram-se 30 pares de rins dessa espécie, obtidos de animais adultos, machos e fêmeas. O material objeto da presente investigação foi obtido junto a criadouro/abatedouro, devidamente autorizado, situado no município de Quedas do Iguaçu, na região Sudoeste do estado do Paraná. Após coletados os rins, que foram mantidos aos pares e identificados, canulavam-se as artérias renais, direita e esquerda, procedendo-se a injeção, em 15 pares, com solução corada de Neoprene Látex "450", 1 visando facilitar o reconhecimento, durante a dissecação, do padrão de divisão da artéria renal, para cada um daqueles órgãos; em seguida, foram os mesmos mantidos em solução aquosa de formol a $10 \%$, para sua fixação. Em outros 15 pares de rins, foi injetada substância sintética corada, Vinilite ${ }^{2}$ diluído em Acetona P. A., pela mesma via arterial já descrita, em seguida submetidos ao processo de corrosão ácida (ácido sulfúrico 30\%), visando a obtenção de moldes vasculares arteriais renais.

Tais procedimentos possibilitaram observações e análises, apoiadas por esquematizações e fotografias, viabilizando, assim, o reconhecimento do padrão vascular arterial dos rins na espécie em apreço. Vale ressaltar, no entanto, a preocupação do presente trabalho em esclarecer a forma de divisão primária das artérias renais, cujos ramos definem a divisão do órgão em setores vasculares (ramos setoriais), bem como as suas subdivisões em ramos interlobares, destinados aos segmentos renais (ramos segmentares).

\section{Resultados}

Ao investigarem-se as divisões e subdivisões das artérias renais, direita e esquerda, em queixadas (Tayassu pecari), verificou-se que a primeira delas se dá, à direita, ao nível pré-hilar em $73,3 \%$ das observações e hilar nos demais $26,6 \%$; porém, à esquerda, ocorre ao nível pré-hilar em $70,0 \%$ e ao nível hilar nos $30,0 \%$ restantes, além do que a seguir se expõe:

I. Da divisão primária, em ramos setoriais, das artérias renais, resultaram:

a) a artéria renal direita dividiu-se em dois ramos setoriais, dorsal e ventral, em 11 casos estudados $(36,6 \%)$; em três ramos, ventral, dorsocranial e dorsocaudal, em outros 11 casos (36,6\%); ainda por bifurcação, agora originando ramos cranial e caudal, em cinco animais $(16,6 \%)$; ou por trifurcação, em ramos dorsal, ventrocranial e ventrocaudal, disposição encontrada em três espécimes $(10,0 \%)$;

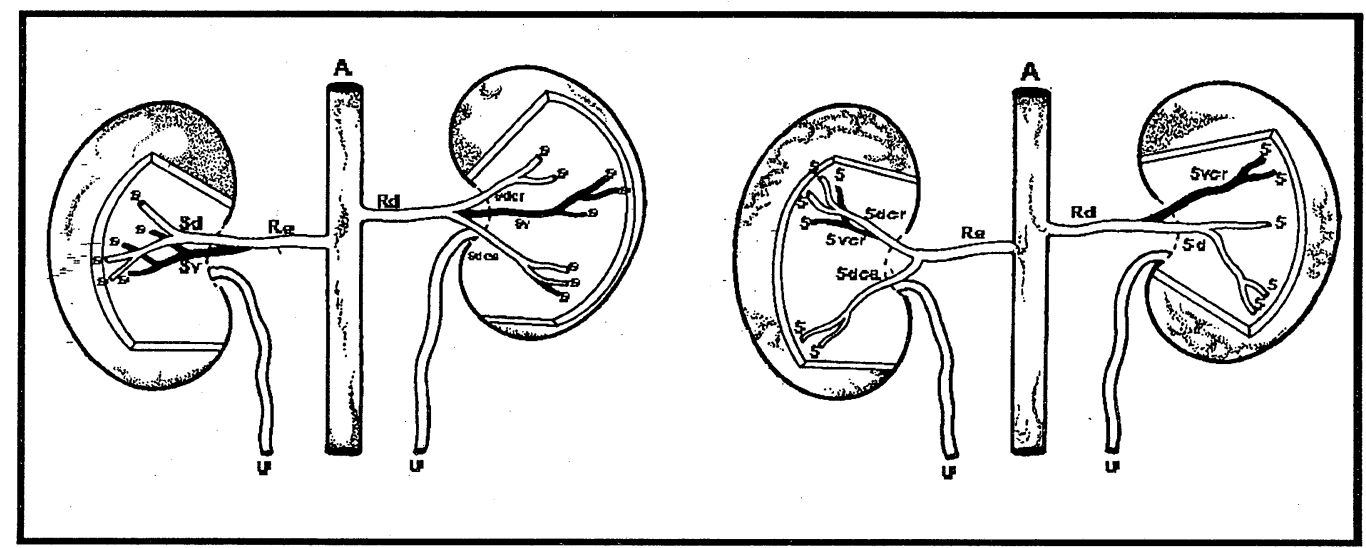

Figura 1: Representação esquemática alusiva a duas formas de divisões arteriais renais, em queixadas (Tayassu pecari). Aorta (A); artéria renal direita (Rd); artéria renal esquerda (Re); ramo setorial dorsal (Sd); ramo setorial ventral (Sv); ramo setorial dorsocranial (Sdcr); ramo setorial dorsocaudal (Sdca); ramo setorial ventral (Sv); ramo setorial ventrocranial (Svcr); ramos segmentares (S); ureteres (U). Palotina, 1999.

\footnotetext{
1 Dupont do Brasil S/A.

${ }^{2}$ Bakelite vinyl - Bland VMCH, Union Carbide Corporation - Chemical and Plastic, N. Y. - USA.
} 
b) a artéria renal esquerda emitiu três ramos setoriais em 11 oportunidades $(36,6 \%)$, sendo esses ramos ventral, dorsocranial e dorsocaudal; dividiu-se por bifurcação, em ramos dorsal e ventral, vistos em 10 casos (33,3\%); ainda por bifurcação, agora dando ramos cranial e caudal, em cinco ocasiões (16,6\%); trifurcou-se, ainda, em ramos dorsal, ventrocranial e ventrocaudal, em quatro oportunidades $(13,3 \%)$.

II. Da segunda divisão das artérias renais surgem ramos interlobares, destinados aos segmentos renais, por este motivo aqui identificados por ramos segmentares, como se segue:

a) no rim direito, o ramo setorial dorsal apareceu 14 vezes $(46,6 \%)$, emitindo, em todos esses casos, três ramos segmentares; o ramo setorial ventral, observado 22 vezes $(73,3 \%)$, enviou três ramos segmentares em 16 casos $(53,3 \%)$ e dois daqueles ramos nos demais seis casos $(20,0 \%)$; o ramo setorial dorsocranial, que foi encontrado em 11 dissecações $(36,6 \%)$, enviou dois ramos segmentares em nove delas $(30,0 \%)$ e três ramos nas duas outras oportunidades $(6,66 \%)$; o ramo setorial dorsocaudal, observado em 11 ocasiões $(36,6 \%)$, dividiu-se em dois ramos segmentares em 10 delas $(33,3 \%)$ e, na última $(3,33 \%)$, dividiu-se em três ramos segmentares; o ramo setorial cranial, presente em cinco oportunidades (16,6\%), trifurcou-se em três delas $(10,0 \%)$, bifurcando-se nas duas outras $(6,66 \%)$, originando, assim, três e dois ramos segmentares, respectivamente; já o ramo setorial caudal, presente em cinco ocasiões (16,6\%), dividiu-se em três delas $(10,0 \%)$, dando três ramos segmentares, sendo que nas duas restantes $(6,66 \%)$ emitiu dois ramos segmentares; o ramo setorial ventrocranial, nas ocasiões em que foi visto, três vezes $(10,0 \%)$, originou dois ramos segmentares; já a artéria setorial ventrocaudal, quando presente em três ocasiões $(10,0 \%)$, originou dois ramos segmentares duas vezes $(6,66 \%)$ e três daqueles ramos em uma oportunidade.

b) no rim esquerdo, o ramo setorial dorsal, presente em 14 dissecações $(46,6 \%)$, emitiu três ramos segmentares em nove delas $(30,0 \%)$ e dois daqueles ramos nas cinco outras ocasiões $(16,6 \%)$; o ramo setorial ventral, encontrado em 21 animais $(70,0 \%)$, dividiu-se por trifurcação em oito deles $(26,6 \%)$, bifurcando-se nos 13 casos restantes $(43,3 \%)$; o ramo setorial cranial, encontrado cinco vezes $(16,6 \%)$ no rim esquerdo, dividiu-se em dois ramos segmentares em todas elas; o ramo setorial caudal, presente em cinco oportunidades (16,6\%), emitiu dois ramos segmentares em todas as ocasiões; o ramo setorial dorsocranial, encontrado 11 vezes $(36,6 \%)$, dividiu-se em três ramos segmentares em sete delas $(23,3 \%)$. Emitiu, porém, em quatro oportunidades $(13,3 \%)$, apenas dois daqueles ramos; já o ramo setorial dorsocaudal, também reconhecido em 11 oportunidades $(36,6 \%)$, emitiu dois ramos segmentares em nove oportunidades $(30,0 \%)$ e três daqueles ramos nas duas outras ocasiões $(6,66 \%)$; o ramo setorial ventrocranial, presente em três espécimes estudados $(10,0 \%)$, dividiu-se em três ramos em todos eles; já o ramo setorial ventrocaudal, também encontrado em três ocasiões $(10,0 \%)$, emitiu, em todas elas, dois ramos segmentares.

\section{Discussão}

Ao analisarem-se os achados do presente trabalho, torna-se evidente a impossibilidade de sua confrontação com aqueles apresentados por outros autores, sem dúvida discrepantes, principalmente por tratarem de espécies distintas.

Entretanto, vale lembrar, autores como Caradonna (1930), Favilli (1931) e Bossi (s.d.), referindo-se ao cavalo, ressaltam as divisões da artéria renal em dois ou três ramos, sem especificar se diretos ou indiretos; porém, em sendo diretos, caberia comparação aos ramos setoriais aludidos no presente trabalho. Já Ellemberger e Baum (1932) destacam a presença de quatro ou cinco ramos, remetendo à questão se seriam diretos ou indiretos. Tal assertiva também se aplica às informações coIhidas em Lesbre (1923), Bruni, Zimmerl (1947). Schummer, Nickel (1960), Schwarze, Schröder (1962), Dobberstein, Hoffmann (1963), ao afirmarem que a artéria renal atinge o hilo ainda indivisa, contrariam os presentes achados, que registram, em cerca de $70 \%$ dos casos, a divisão primária das artérias renais em situação pré-hilar. Os dados de Miglino et al. (1985), embora obtidos em carneiros, contemplam alguns aspectos do presente trabalho. Observações que também se aplicam às informações exaradas por El-Khaligi et al. (1987).

No tocante às informações referentes à divisão primária das artérias renais, Peduti-Neto (1976), Albuquerque (1979), Miglino et al. (1985), Carvalho, Pereira (1987), Carvalho (1995), referindo-se a pequenos ruminantes, confirmam a presença dos ramos (setoriais) dorsal e ventral, referendados no presente trabalho em apenas $36,6 \%$ dos casos. Entretanto, ainda nesse aspecto, vale a ressalva dos achados de Ferreira (1980), Fagundes (1984), Sampaio et al. (1996), Evan et al. (1996) e Souza (1997), referentes ao suíno, que consideram o predomínio dos ramos (setoriais) cranial e caudal. Dados esses confirmados no presente estudo, porém, sem o predomínio ressaltado por aqueles autores, tendo sido encontrados, no queixada, em $16,6 \%$ dos casos investigados. Os presentes achados, no que tange aos aspectos da divisão primária das artérias renais, colocam o queixada em situação diferenciada em relação às espécies acima referidas.

Faz-se oportuno o destaque da impropriedade de maiores confrontos dos resultados expressos no presente trabalho com aqueles acima referidos, não só por tratarem-se de espécies distintas, mas, também, pelos diferentes objetivos propostos: reportar-se apenas aos aspectos da divisão primária das artérias renais (artérias setoriais) e seu desdobramento em artérias segmentares, ficando a abordagem de sua responsabilidade territorial, com vistas ao, estudo da segmentação, para futuras descrições.

\section{Conclusões}

Em face dos resultados obtidos do estudo de 30 pares de rins de queixadas (Tayassu pecarı), machos e fêmeas, adultos, julga-se poder concluir que:

a) as artérias renais dividem-se, primariamente, em ramos setoriais pré-hilares e hilares;

b) a artéria renal direita emite dois ramos setoriais, dorsal e ventral $(36,6 \%)$, três ramos setoriais, ventral, dorsocranial 
e dorsocaudal $(36,6 \%)$, dois ramos setoriais, cranial e caudal $(16,6 \%)$ ou três ramos setoriais, dorsal, ventrocranial e ventrocaudal $(10,0 \%)$.

c) a artéria renal esquerda divide-se em três ramos setoriais - ventral, dorsocranial e dorsocaudal $(36,6 \%)$; em dois ramos setoriais - dorsal e ventral $(33,3 \%)$; em dois ramos setoriais - cranial e caudal $(16,6 \%)$, ou três ramos setoriais - dorsal, ventrocranial e ventrocaudal (13,3\%);

\section{Referências bibliográficas}

ALBUQUERQUE, J F C. Contribuição ao estudo da vascularização arterial do rim em caprinos (Capra hircus Linnaeus, 1758). 1979. 60 p. Tese (Doutorado) - Faculdade de Medicina Veterinária e Zootecnia da Universidade de São Paulo, São Paulo.

BOSSI, V., CARADONNA, G. B., SPAMPANI, G., VARALDI, L., ZIMMERL, $U$ Trattato di anatomia veterinaria. Milano: Francesco Vallardi, (s.d.). v. 2, p. 811.

BRUNI, A. C., ZIMMERL, U. Anatomia degli animali domestici. Milano: Francesco Vallardi, 1947, v. 2, p. 156.

CARADONNA, G. B. In: ZIMMERL, U. Trattato di anatomia veterinaria. Milano : Francesco Vallardi, 1930, v. 3, p. 23.

CARVALHO, M. A. M. Segmentos anatomocirúrgicos arteriais do rim de caprinos. 1995. 152 p. Tese (Doutorado) - Universidade de São Paulo, São Paulo.

CARVALHO, M. A. M., PEREIRA, J. G. L. Contribuição ao estudo dos elementos vasculares, arteriais e venosos, hilares e extra-hilares, em rins de caprinos da raça Moxotó. Rev Fac Med Vet Zoot Univ São Paulo, v. 24, n. 2, p. 119-126. 1987.

DIDIO, L. J. A. Segments of the kidney. The anatomical basis for nephrosegmentectomy. In: Didio L J A, Motta P. Basic clinical, and surgical nephrology. Boston: Martins Nijhoff , 1985. p. 1-12.

DOBBERSTEIN, J., HOFFMANN, G. Lerbuch der vergleichenden Anatomie der Haustiere. Leipzig: S. Hirzel, 1963, v. 1, p. 136.

ELLEMBERGER, W., BAUM, H. Handbuch der Vergleichenden Anatomie der Haustiere. Berlin : Julius Springer, 1932. p. 528.

EL-KHALIGI, G., M., OSNAN, F. A., ABU-ZAID, S., EL-NAHLA, S. Gross anatomical studies on the renal arteries of some domestic animals. Part II: Sheep. Vet Med Jour, v. 35, n. 1, p. 7-15, 1987.

EVAN, A. P., CONNORS, B. A., LINGEMAN, J. E., BLOMGREN, P., WILLIS, L. R. Branching patterns of the renal artery of the pig. Anat Rev, v. 246, p. 217-223, 1996.

FAGUNDES, G. M. 1984. Contribuição ao estudo da vascularização arterial do rim em suínos (sus scrofa domestica Linnaeus, 1758). d) os ramos setoriais - oriundos da divisão primária das artérias renais, emitem de dois a três ramos segmentares, destinados a territórios próprios do parênquima renal;

e) a análise dos resultados não evidenciou diferença estatisticamente significativa, ao nível de $5,0 \%$, relativamente ao sexo, quando confrontados o número e a distribuição arterial nos rins investigados.

1984. 82 p. Tese (Doutorado) - Faculdade de Medicina Veterinária e Zootecnia da Universidade de São Paulo.

FAVILLI, N. Nozioni comparate di anatomia e fisiologia degli animali rurali. Torino : Editrice Torinese, 1931, p. 451.

FERREIRA, N. Contribuição ao estudo dos elementos vasculares, arteriais e venosos, do hilo renal em suínos (sus scrofa domestica). Rev Fac Med Vet Zoot Univ São Paulo, v. 12, p. 7-22, 1975.

FOWLER, M. E. Zoo \& wild animal medicine - Current therapy. Philadelphia: WB Saunders, 1986. p. 513-522.

KILTIE, R. A. Stomach contents of rain forest peccaries (Tayassuidae). Biotropica, v. 13, n. 3, p. 234-236, 1981.

LANGER, P. Stomach evolution in the Artiodactila. Mammalia, v. 38, n. 2, p. 127-136, 1974.

LESBRE, F. X. Précis d'anatomie comparée des animaux domestiques. Paris : J. B. Baillière et Fills, 1923. V. 2, p. 374.

MIGLINO, M. A., SOUZA, W. M., CARVALHO M. A. M., MARIANA, A. N. B. Sobre a vascularização renal de ovinos (Ovis aries Linnaeus, 1758) da raça Ideal. CONGRESSO BRASILEIRO DE ANATOMIA, 14., 1985. Vitória. Anais... p. 86.

ORR, R. T. Biologia dos vertebrados. San Francisco : Academy of Sciences, 1986. p. 242.

PEDUTI-NETO, J. Contribuição ao estudo dos elementos vasculares, arteriais e venosos, do hilo renal, em ovinos da raça Merino. Rev Fac Med Vet Zoot Univ São Paulo, v. 13, n. 1, p. 67-83, 1976.

SAMPAIO, M. A. P. P., ALBUQUERQUE, L. F. P., SAMPAIO, F. J. B. Intrarenal arterial distribution in swine. CONGRESSO BRASILEIRO DE ANATOMIA, 17., 1996. Fortaleza. Anais... p. 93.

SCHUMMER, A., NICKEL, R. In: NICKEL, R., SCHUMMER, A., SEIFERLE, E. 1960. Lehrbuch der Anatomie der Haustiere. Berlin: Paul Parey, V. 2, p. 300.

SCHWARZE, E., SCHRÖDER, L. Kompendium derveterinär Anatomie. Jena: Gustav Ficherv, 1962. V. 2, p. 241.

SOUZA, N. T. M. Segmentos anatomocirúrgicos arteriais do rim em suínos (sus scrofa domestica Linnaeus, 1758). 1997. 104 p. Tese (Doutorado) - Faculdade de Medicina Veterinária e Zootecnia da Universidade de São Paulo, São Paulo. 